\title{
Still making progress
}

\author{
Marija Balic
}

Received: 13 August 2018 / Accepted: 13 August 2018

(c) Springer-Verlag GmbH Austria, part of Springer Nature 2018

Although the outcome of breast cancer patients has improved over the last few decades, especially for advanced disease, there is still a need for novel treatment strategies. The field of medical oncology has been lately transformed, and a variety of new treatment options have been approved for many indications, with greatest improvement generated by immunotherapy. Breast cancer treatment has paved the way for development of stratification strategies and individualization of treatment. Recently, however, greater progress has been in systemic therapies for other metastatic cancers.

At the 40th San Antonio Breast Cancer Symposium 2017 there were several preclinical and clinical studies establishing further biological insights for development of novel treatments and novel clinical data potentially influencing daily practice in the treatment of breast cancer patients.

Some preclinical data were shown on selective estrogen receptor covalent antagonists (SERCAs). Immune-modulating effects have gained importance, and preliminary clinical studies with immunotherapeutic strategies are promising, and it should not take long until immune checkpoint inhibitors are approved for the treatment of selected advanced breast cancer patients. Novel technology improvements enable better biological characterization and molecular understanding of intratumoral heterogeneity of breast cancer subgroups. Zsuzsana Bago-Horvath [1] summarizes the progress of translational studies presented at the 40th San Antonio Breast Cancer Symposium 2017.

\section{Balic ( $₫)$}

Division of Clinical Oncology, Department of Internal

Medicine, Medical University Graz, Graz, Austria

marija.balic@medunigraz.at
There are still a few important topics waiting for definitive answers in the locoregional treatment and several studies have attempted to elucidate and define standards. Sentinel lymph node determination has long become standard in the primary surgery of localized breast cancer. De-escalation of axillary surgery still remains a debated topic in the neoadjuvant treatment of patients. Of particular importance is the definition of safety of sentinel lymph node determination and de-escalation of surgery in primary clinically positive axilla converting to negative after neoadjuvant therapy. Selected studies on this topic and selected locoregional studies presented at the 40th San Antonio Breast Cancer Symposium 2017 are summarized by Florian Fitzal [2].

Liquid biopsy encompasses circulating tumor cells and cell free tumor specific DNA. Whereas detection and the count of circulating tumor cells were repeatedly shown to be of prognostic significance in advanced breast cancer settings, little data on their significance exist in early stage patients. One large study was presented in San Antonio in 2017. However, greater interest is in detection of cell free tumor specific DNA in the belief that better molecular understanding of disease and metastasis can be provided. Along with liquid biomarkers Rinnerthaler et al. [3] report on selected lifestyle factors. In addition to previously published studies on CDK4/6 inhibitors, first data were shown that the same effect can be demonstrated in premenopausal population. Meta-analysis of older patients treated with CDK4/6 inhibitors was presented, showing the same clinical benefit accompanied by increased toxicity. This has to be taken into account when treating older patients with advanced breast cancer.

With respect to systemic therapy, there is room for further progress even if the achieved steps are getting smaller with better outcome of control arms over 
time. There are still undefined questions regarding chemotherapy schedule and substances, and dosedense therapy in high-risk populations is becoming a standard. With novel antibody drug conjugates especially in aggressive disease impressive results are achieved. And finally, even though immune checkpoint inhibitors are already part of the treatment standard in lung cancer or urological cancers, in breast cancer attractive combinational strategies are being tested in more immunogenic subgroups and these strategies are promising so that we will soon be able to also improve outcome with this approach in breast cancer. Rupert Bartsch [4] has selected the most important studies presented at the 40th San Antonio Breast Cancer Symposium 2017.

San Antonio Breast Cancer Symposium 2017 again demonstrated that clinically important issues are being addressed and the treatment of breast cancer patients is optimized towards the best outcome currently possible at the cost of increasingly little deterioration of patients' life quality.

Conflict of interest M. Balic declares that she has no competing interests.

\section{References}

1. Bago-Horvath Z. SABCS 2017 pathology: from bench to bedside. memo. 2018. https://doi.org/10.1007/s12254018-0427-8.

2. Fitzal F. Post SABCS local therapy and radiology. memo. 2018. https://doi.org/10.1007/s12254-018-0403-3.

3. Rinnerthaler G, Gampenrieder SP, Greil R. SABCS 2017: lifestyle factors, hormone receptor-positive advanced disease, liquid biopsies, and prognosis. memo. 2018. https:// doi.org/10.1007/s12254-018-0433-x.

4. Bartsch R, Bergen E. SABCS 2017: update on chemotherapy, targeted therapy, and immunotherapy. memo. 2018. https://doi.org/10.1007/s12254-018-0430-0.

Marija Balic, Assoc. Professor, Medical Oncologist

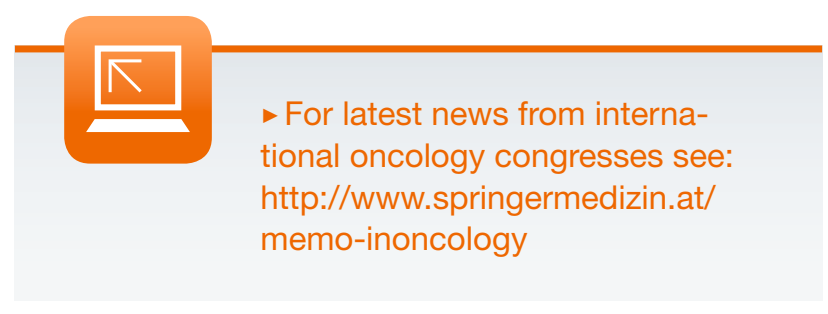

